June, 1994

UM-TH-94-21

UMN-TH-1303-94

FERMILAB-Pub-94/199-A

hep-ph/yymmddd

\title{
Bounds on Dirac Neutrino Masses from Nucleosynthesis
}

\author{
A.D. Dolgov ${ }^{1,2,3}$, K. Kainulainen ${ }^{4}$ and I.Z. Rothstein ${ }^{3}$ \\ ${ }^{1}$ Institute of Theoretical and Experimental Physics, \\ Moscow 117259, Russia, \\ ${ }^{2}$ NASA/Fermilab Astrophysics Center, Fermi National \\ Accelerator Laboratory, Batavia, IL 60510 \\ ${ }^{3}$ Randall Laboratory of Physics, \\ University of Michigan, Ann Arbor, MI 48109-1120 \\ ${ }^{4}$ School of Physics and Astronomy, University of Minnesota \\ Minneapolis, MN 55455, USA
}

\begin{abstract}
We derive new bounds on the Dirac mass of the tau and muonic neutrinos. By solving the kinetic equation for the rate of energy deposition due to helicity flipping processes and imposing the constraint that the number of effective species contributing to the energy density at the time of nucleosynthesis be $\Delta k_{\nu}<0.3$, we find the bounds $m_{\nu_{\mu}}<150 \mathrm{KeV}$ and $m_{\nu_{\tau}}<190 \mathrm{KeV}$ for $T_{\mathrm{QCD}}=200 \mathrm{MeV}$. The constraint $\Delta k_{\nu}<0.1$ leads to the much stronger bound $m_{\nu}<10 \mathrm{KeV}$ for both species of neutrinos.
\end{abstract}


If neutrinos are massive and stable, then the mass range $40 \mathrm{eV}<m_{\nu}<2 \mathrm{GeV}$ [1, 2] is excluded by measurements of the age of the universe. However, if the neutrinos are unstable then these bounds will not apply and we must search elsewhere to find mass restrictions. The present laboratory bounds on the muonic and tau neutrino are $160 \mathrm{KeV}$ [3] and $31 \mathrm{MeV}$ [4] respectively. Better bounds may be had if the lifetime of the massive neutrino is $>\mathcal{O}(100)$ sec. In this range of lifetimes the energy density due to the massive species may come to increase the expansion rate of the universe and thereby increase the primordial helium abundance [5]. In this case the mass ranges $0.5<m<35 \mathrm{MeV}$ and $0.3<m<35 \mathrm{MeV}$ were found to be excluded for Majorana and Dirac neutrinos respectively [6]. The mass bound for Dirac neutrinos was derived assuming that the right handed species is in thermal equilibrium below the QCD phase transition, for $m>300 \mathrm{KeV}$. In this letter we show that even if the right handed neutrinos do not enter thermal equilibrium at temperatures below $T_{Q C D}$, their out-of-equilibrium production rate is strong enough to yield a much more stringent lower bound on the Dirac neutrino mass.

The rate of production of right handed neutrinos is roughly given by, $\Gamma_{-} \propto\left(m_{\nu} / E\right)^{2} G_{F}^{2} T^{5}$ where $E$ is the energy of the neutrino and $\Gamma_{W}$ is the normal weak interaction rate. Thus, the "wrong" helicity states" are produced more efficiently at higher temperatures. If the wrong helicity states decouple from thermal equilibrium above $T_{\mathrm{QCD}}$, their energy density will be greatly diluted in comparison with the coupled species as a consequence of entropy conservation. Such neutrinos would appear to be cosmologically safe. A bound based on the decoupling argument was first derived in ref. [8], where it was shown that the Dirac mass must satisfy $m_{\nu}<300 \mathrm{KeV}$, assuming that the QCD phase transition temperature is 100 $\mathrm{MeV}$. The result of ref. [8], was refined in ref. [7], where a more careful computation of the relevant scattering processes was performed and much weaker bounds were claimed.

In ref. [9] a different bound, $m_{\nu_{\mu}}<420 \mathrm{KeV}$ was obtained by considering the out-ofequilibrium production of wrong helicity states through the pion resonance $\gamma \gamma \rightarrow \pi^{0} \rightarrow \nu_{+} \bar{\nu}_{+}$, and imposing the constraint $\Delta k_{\nu}<0.3$. Unlike the bound discussed first above, this result is insensitive to $T_{\mathrm{QCD}}$, as will be shown below.

\footnotetext{
${ }^{1}$ In the rest of the paper, when we refer to "wrong" helicity states, we mean right handed neutrinos and left handed anti-neutrinos.
} 
In this letter we give a more accurate analysis of the production of light wrong helicity states in the early universe. We will account for all sources of wrong helicity neutrinos, including the population that decoupled above $T_{\mathrm{QCD}}$ as well as those generated from the following out-of-equilibrium processes:

$$
\begin{aligned}
\pi^{0} & \rightarrow \nu_{\mu(\tau)+} \bar{\nu}_{\mu(\tau)+} \\
\pi^{+} & \rightarrow \mu^{+} \nu_{\mu+} \\
l_{1} l_{2} & \rightarrow l_{3} \nu_{\mu(\tau)+} \\
l \bar{l} & \rightarrow \nu_{\mu(\tau)+} \bar{\nu}_{\mu(\tau)+} .
\end{aligned}
$$

Moreover, we will accurately solve the kinetic equation for neutrino energy density generated out-of-equilibrium at temperatures below the QCD phase transition. Our bounds will be both more precise and much stronger than any found in earlier literature.

We should mention here that strong bounds on the neutrino mass, $m_{\nu}<10-20 \mathrm{KeV}$, were obtained from the consideration of the cooling of supernova SN-87 [10, [1]. While these bounds are indeed more stringent than the bounds obtained in this paper, they are in a sense model dependent. Supernova bounds come from calculating the rate at which energy is drained from the supernova assuming that the right handed neutrinos are sterile and will free stream from the core. However, since the right handed neutrinos are singlets, it is quite possible that they interact in yet unknown ways [12]. F Furthermore, with better data it may be possible that the nucleosynthesis bounds will eventually become more stringent than those obtained from supernova considerations.

We will first consider helicity flipping scatterings $\nu_{\mu(\tau)-} l \rightarrow \nu_{\mu(\tau)+} l$, and annihilations $l \bar{l} \rightarrow \nu_{\mu(\tau)+} \bar{\nu}_{\mu(\tau)+}$ where $l$ is a lepton (not the tau however) and sub- \pm refers to the helicity of the neutrino. The amplitude for $t$-channel elastic scattering process is given by

$$
A\left(\nu l ; \nu_{+} l\right)=\frac{G_{F}}{\sqrt{2}} \bar{\nu} \gamma_{\alpha}\left(1-\gamma_{5}\right) \nu_{+} \bar{l}\left(c_{V}-c_{A} \gamma_{5}\right) \gamma_{\alpha} l,
$$

\footnotetext{
${ }^{2}$ If the right handed species is trapped inside supernova, thus obviating the bound derived in ref. [10, 11, it would likely contribute to the energy density at the time of nucleosynthesis. To avoid this, physics far beyond the standard model is needed [13].
} 
where, for example in $\nu \nu$-scattering $c_{V}=c_{A}=\frac{1}{2}$ and in $\nu e$-scattering $c_{A}=2 \sin ^{2} \theta_{W}-\frac{1}{2}$ and $c_{V}=\frac{1}{2}$. The projection onto the wrong helicity state is accomplished by using

$$
\sum_{\text {spins }} \nu_{+} \bar{\nu}_{-}=\frac{1}{2}\left(\not k^{\prime}+m_{\nu}\right)\left(1+\gamma_{5} \hat{s}\right)
$$

where the four-vector $s$ is given by the expression:

$$
s=\frac{\lambda}{m_{\nu}}\left(k^{\prime}, \omega^{\prime} \vec{n}^{\prime}\right)
$$

Here the helicity eigenvalue $\lambda= \pm 1$ determines the helicity state of final state neutrino, $k^{\prime}=$ $\left|\overrightarrow{k^{\prime}}\right|$ is the magnitude of the spatial component of the neutrino momentum, $\omega^{\prime}=\left(k^{\prime 2}+m_{\nu}^{2}\right)^{1 / 2}$ is its energy, and $\vec{n}$ is the unit vector in direction of $\overrightarrow{k^{\prime}}$.

The amplitude squared for the production of the neutrinos with the wrong helicity in elastic scattering is

$$
\begin{aligned}
\left|A\left(\nu l ; \nu_{+} l\right)\right|^{2}=16 G_{F}^{2} & \left\{\left(c_{V} \pm c_{A}\right)^{2}(p \cdot k)\left(p^{\prime} \cdot l\right)\right. \\
+ & \left.\left(c_{V} \mp c_{A}\right)^{2}\left(p^{\prime} \cdot k\right)(p \cdot l)-\left(c_{V}^{2}-c_{A}^{2}\right) m_{l}^{2}(k \cdot l)\right\}
\end{aligned}
$$

where $p, p^{\prime}$, are the four-momenta of the initial and final fermions, and $k, k^{\prime}$ are the momenta of the neutrinos $\nu_{-}$and $\nu_{+}$respectively. The upper (lower) signs refer to scattering off particles (antiparticles) and we defined the four-vector $l=\left(\omega^{\prime}-k^{\prime}\right)(1,-\vec{n})=\left(m_{\nu}^{2} / 2 k^{\prime}\right)(1,-\vec{n})$. The amplitude for annihilation process may be obtained from (5) by simple crossing relation. One may see that for the scattering off of neutrinos, $|A|^{2}$ goes like the neutrino mass to the fourth power in the center of mass frame. It is because of this seemingly slow rate that these processes were neglected in ref. [7]. However, this suppression is not present in an arbitrary frame and generically the production of the wrong helicity neutrinos is suppressed only as $m_{\nu}^{2}$. This is a consequence of the fact that the helicity is not a Lorentz invariant quantity. Indeed, given a particle with definite negative helicity, a boost in a direction orthogonal to its direction of motion with velocity $v$ will generate an admixture of the positive helicity equal to $m^{2} v^{2} / 4 E^{2}$.

The kinetic equation for the energy density of the right-handed neutrinos has the form

$$
\begin{aligned}
\frac{d \rho_{\nu}}{d t}+4 H \rho_{\nu} \equiv C_{t}=\frac{1}{(2 \pi)^{8}} & \int \frac{d^{3} p}{2 E} \frac{d^{3} k}{2 \omega} \frac{d^{3} p^{\prime}}{2 E^{\prime}} \frac{d^{3} k^{\prime}}{2 \omega^{\prime}} \omega^{\prime} \delta^{4}\left(p+k-p^{\prime}-k^{\prime}\right) \times \\
& \times\left\{\left|A_{t}\right|^{2} f_{\nu}(\omega) f_{l}(E)\left[1-f_{l}\left(E^{\prime}\right)\right]+\ldots\right\},
\end{aligned}
$$


where the ellipses refer to the remaining elastic scattering (off antiparticles) and annihilation channels as well as to the contributions from the pion resonances which will be treated below. Note that we omitted the Pauli blocking factor for wrong helicity states, which are not in thermal equilibrium. We also neglected the inverse reaction of right-handed neutrinos producing the normal ones. This is justified because the density of the wrong neutrinos has to be small. For the same reason we can compute all contributions to $\rho_{\nu}$ separately and add the different contributions in the end.

If we assume that the phase space distribution functions are of the equilibrium Boltzmann form, $f=\exp (-E / T)$, we can compute the collision integral on the r.h.s. of equation (6) analytically in the massless limit. For example, the matrix element for a $t$-channel reaction in (5) gives

$$
C_{t}\left(m_{f}=0\right)_{M B}=\frac{G_{F}^{2} m_{\nu}^{2} T^{7}}{16 \pi^{5}}\left\{\left(c_{V}+c_{A}\right)^{2}+\frac{5}{6}\left(c_{V}-c_{A}\right)^{2}\right\}
$$

We have also computed the collision integrals numerically retaining the effect Fermi statistics, and found a $21 \%$ suppression relative to the case of Boltzmann statistics. Furthermore, it was found that this suppression is nearly the same for all the reactions.

We took into account the following scattering processes: $\nu_{\mu-} \nu_{e} \rightarrow \nu_{\mu+} \nu_{e}, \nu_{\mu-} \nu_{\mu} \rightarrow$ $\nu_{\mu+} \nu_{\mu}, \nu_{\mu-} \nu_{\tau} \rightarrow \nu_{\mu+} \nu_{\tau}, \nu_{\mu-} e^{-} \rightarrow \nu_{\mu+} e^{-}, \nu_{\mu-} \mu^{-} \rightarrow \nu_{\mu+} \mu^{-}$, with the corresponding channels for scattering off antiparticles and the annihilation channels. Similar channels exist also for $\nu_{\tau+}$-production. However, the reaction channels involving muons are different, due to the contribution from charged current scattering. Moreover, in the muonic case there are three more pure charged current channels that do not exist for $\nu_{\tau+-}$-production: $\mu^{-} \nu_{e} \rightarrow$ $\nu_{\mu+} e^{-}, \mu^{-} e^{+} \rightarrow \nu_{\mu+} \overline{\nu_{e}}$ and $e^{+} \nu_{e} \rightarrow \nu_{\mu+} \mu^{+}$. There is also a contribution from scattering off of pions, which we found to be small and will be neglected.

Equation (7), modified to include Fermi statistics, can be used for all relevant reaction channels except for those involving muons. For the muonic channels we computed the collision integrals numerically as a function of temperature. Altogether we can write the kinetic equation as

$$
\dot{\rho}_{\nu}+4 H \rho_{\nu}=\frac{G_{F}^{2} m_{\nu}^{2}}{2 \pi^{5}} c_{\mathrm{F}} N_{\mathrm{eff}} T^{7},
$$

where the Fermi correction factor $c_{\mathrm{F}} \simeq 0.79$ and the effective number of reactions $N_{\text {eff }}$ 
(normalized such that $t$-channel scattering off neutrinos gives a contribution 1), for example in $\nu_{\mu}$-case is given by

$$
N_{\mathrm{eff}, \mu}=\left(\frac{16}{3}\right) \cdot\left\{1+\sum_{f=e, \nu_{e}, \nu_{\tau}}\left(c_{V f}^{2}+c_{A f}^{2}\right)+\left(c_{V \mu}^{2}+c_{A \mu}^{2}+2\right) d_{\mu}(T)\right\} .
$$

The first '1' comes from scattering off of muonic neutrinos and $c_{V \mu}=2 \sin ^{2} \theta_{W}+\frac{1}{2}, c_{A \mu}=\frac{1}{2}$ (see also below equation (2)). Using $\sin ^{2} \theta_{W}=0.23$, we find

$$
\begin{aligned}
& N_{\mathrm{eff}, \mu} \simeq 3.92\left(1+0.81 d_{\mu}(T)\right) \\
& N_{\mathrm{eff}, \tau} \simeq 3.92\left(1+0.06 d_{\tau}(T)\right)
\end{aligned}
$$

where the fit functions $d_{i}(T)$ are normalized to become unity for very large $T$. The suppression due to the muon mass becomes significant rather late: for $T=m_{\mu} / 3$ we still get $d_{\mu} \simeq 0.42$ and $d_{\tau} \simeq 0.35$. Hence the muonic interactions are important in deriving the mass bounds. For $T=m_{\mu} / 10$ however, we find $d_{\mu} \simeq 4.7 \times 10^{-3}$, which clearly validates our neglect of reactions involving $\tau \mathrm{s}$.

If all the particles in the plasma were massless we could easily integrate the equations (8-10) since in this case the temperature and the time are related as $T^{2} t=$ const. However, since the contribution of massive pions and muons to the energy density is essential, we should proceed more carefully. If we neglect the tiny contribution to the pressure density $p$ coming from the wrong helicity states, the covariant energy conservation law $\dot{\rho}=-3 H(\rho+p)$ reduces to the usual conservation of entropy of interacting species, and we get the standard relation between time and temperature [14

$$
\frac{d t}{d T}=-\left(\frac{45}{4 \pi^{3}}\right)^{1 / 2} \frac{m_{P l}}{T^{3}} \frac{1}{g_{*}^{1 / 2}(T)}\left(1+\frac{T}{3 h_{*}} \frac{d h_{*}}{d T}\right)
$$

Here $g_{*}$ and $h_{*}$ are the effective numbers of energy and entropy degrees of freedom, defined through the total energy density $\rho \equiv \frac{\pi^{2}}{30} g_{*}(T) T^{4}$ and the entropy density of interacting species $s_{I} \equiv \frac{2 \pi^{2}}{45} h_{*}(T) T^{3}$.

Equations (8-11) are easily solved to yield a simple integral expression for the relative energy density $r_{1} \equiv \rho_{\nu+} / \rho_{\nu-}=\rho_{\nu+} /\left(7 \pi^{2} T^{4} / 240\right)$ :

$$
r_{1}=2.88\left(\frac{m_{\nu}}{\mathrm{MeV}}\right)^{2} \int_{0}^{T_{\mathrm{QCD}}} \mathrm{d} T\left(\frac{10.75}{h_{*}(T)}\right)^{4 / 3}\left(\frac{10.75}{g_{*}(T)}\right)^{1 / 2}\left(1+\frac{T}{3 h_{*}} \frac{d h_{*}}{d T}\right)\left(1+a_{i} d_{i}(T)\right) .
$$


Sub-1 means that we take into account only weak scatterings; pion decays are treated separately below. $T_{\mathrm{QCD}}$ is the temperature of the QCD phase transition and the coefficients $a_{i}$ can be read off the equation (10), $a_{\mu}=0.81$ and $a_{\tau}=0.06$. Equation (12) incorporates the effect of the changing energy density due to annihilations of heavy particles, as well as the statement of entropy conservation. We have integrated (12) numerically and found that (for $\left.T_{\mathrm{QCD}} \gtrsim 100\right)$ to a very good accuracy $r_{i}$ s follow the simple linear fits

$$
\begin{aligned}
& r_{1 \mu}=\left(0.53+2.25 T_{\mathrm{QCD}}^{100}\right)\left(m_{\nu} / \mathrm{MeV}\right) \\
& r_{1 \tau}=\left(0.08+1.34 T_{\mathrm{QCD}}^{100}\right)\left(m_{\nu} / \mathrm{MeV}\right)
\end{aligned}
$$

where $T_{\mathrm{QCD}}^{100}$ is the QCD phase transition temperature in units $100 \mathrm{MeV}$. A word of caution is in order here: In equation (12) we assumed that $g_{*}$ and $h_{*}$ reach a constant value 17.25 well above pion mass. This indeed seems to be the correct choice [14], but should they instead increase significantly at temperatures between $m_{\pi}$ and $T_{\mathrm{QCD}}$, then equations (13) would slightly overestimate $r_{1}$ for $T_{\mathrm{QCD}} \gtrsim \mathcal{O}(200) \mathrm{MeV}$.

The decays $\pi^{0} \rightarrow \nu \bar{\nu}$ and $\pi^{ \pm} \rightarrow \mu \nu_{\mu}$ also give significant contributions to the energy density of right-handed neutrinos. The decay of $\pi^{0}$ produces both $\nu_{\mu}$ and $\nu_{\tau}$ while $\pi^{ \pm}$ produces only $\nu_{\mu}$. The former was considered in ref. [9] while the latter, to best of our knowledge, has not been accounted for to date. Both of these processes have the property that their contribution to the energy density is insensitive to the temperature of the QCD transition because the dominant contribution to production of the right-handed $\nu$ occurs at $T \approx m_{\pi} / 5$. Let us first consider the decay $\pi^{0} \rightarrow \nu_{+} \bar{\nu}_{+}$. This width was calculated in ref. [15, 16] as

$$
\Gamma\left(\pi^{0} \rightarrow \nu_{+} \bar{\nu}_{+}\right)=G_{F}^{2} f_{\pi^{0}}^{2} m_{\nu}^{2} m_{\pi} / 8 \pi
$$

where $f_{\pi^{0}} \simeq 93 \mathrm{MeV}$. When computing the $\pi^{0}$ collision term, we will assume the MaxwellBoltzmann statistics. This is an excellent approximation here and moreover we are able to compute the collision term analytically. We find that the energy density of neutrinos produced in $\pi^{0}$-decays satisfies the equation

$$
\dot{\rho}_{\nu}+4 H \rho_{\nu}=\frac{G_{F}^{2} m_{\nu}^{2}}{32 \pi^{3}} f_{\pi^{0}}^{2} T^{5} x_{0}^{4} K_{2}\left(x_{0}\right),
$$


where $x_{0} \equiv m_{\pi^{0}} / T$ and $K_{2}(x)$ is the usual modified Bessel function. This equation can be integrated analogously to the scattering contribution (cf. equation (12)) with the result

$$
r_{2}=1.21\left(m_{\nu} / \mathrm{MeV}\right)^{2},
$$

which to the given accuracy is independent of $T_{\mathrm{QCD}}$. Quite similarly we find for the decay $\pi^{+} \rightarrow \mu^{+} \nu_{+}$:

$$
\dot{\rho}_{\nu}+4 H \rho_{\nu}=\frac{G_{F}^{2} m_{\nu}^{2}}{32 \pi^{3}} f_{\pi^{ \pm}}^{2} T^{5} x_{+}^{4}\left(1-y^{2}\right)\left\{\left(1+y^{2}\right) K_{2}\left(x_{+}\right)-y^{2} K_{0}\left(x_{+}\right)\right\},
$$

where $f_{\pi^{ \pm}} \simeq 128.4, x_{+} \equiv m_{\pi^{ \pm}} / T$ and $y \equiv m_{\mu} / m_{\pi^{ \pm}}$. When integrated, (17) gives

$$
r_{3}=1.15\left(m_{\nu} / \mathrm{MeV}\right)^{2} .
$$

At temperatures above the QCD phase transition, the plasma consisted of free quarks and gluons in addition to leptons and photons and the effective number of degrees of freedom $g_{*}$ was slightly below 60 . Since the efficiency of production of right-handed neutrinos is larger at higher temperatures (at least up to the temperatures of the order of the $W$ and $Z$ boson masses) we expect them to be in thermal equilibrium if their mass is above $\mathcal{O}(10) \mathrm{KeV}$. The energy density of these neutrinos will be diluted in the course of QCD-phase transition and due to annihilation of massive states in the plasma. Using the entropy conservation we may estimate their relative energy density as

$$
r_{\mathrm{QCD}}=(10.75 / 60)^{4 / 3} \simeq 0.10 .
$$

There is one additional effect that needs to be taken into account. Namely, the fact that energy density of a massive species will scale differently than that of a massless species. Thus, the there will be additional contributions to the relative energy density coming from population of left handed neutrinos as well as the small population of right handed neutrinos. Therefore, the total relative energy density may be written as

$$
r_{\text {tot }, \nu}=f\left(m_{\nu}\right)+\left(\sum_{i} r_{i, \nu}+0.1\right)\left(1+f\left(m_{\nu}\right)\right) .
$$

The function $f\left(m_{\nu}\right)$ was found using the results from paper [6], and is given by

$$
f\left(m_{\nu}\right)=1.31 m_{\nu}^{2}+4.84 m_{\nu}^{4}-4.57 m_{\nu}^{6} \equiv 1.31 m_{\nu}^{2}+\delta f,
$$


and $m_{\nu}$ is given in units of $\mathrm{MeV}$. In this way we find the bounds

$$
\begin{aligned}
& m_{\nu_{\mu}}<\left(\frac{\Delta k_{\nu}^{\max }-0.10-\delta_{\mu}}{4.33+2.25 T_{\mathrm{QCD}}^{100}}\right)^{1 / 2} \mathrm{MeV} \\
& m_{\nu_{\tau}}<\left(\frac{\Delta k_{\nu}^{\max }-0.10-\delta_{\tau}}{2.73+1.34 T_{\mathrm{QCD}}^{100}}\right)^{1 / 2} \mathrm{MeV}
\end{aligned}
$$

where $\delta_{\mu}=1.10 \delta f+\left(2.89+2.25 T_{\mathrm{QCD}}^{100}\right) f$ and $\delta_{\tau}=1.10 \delta f+\left(1.29+1.34 T_{\mathrm{QCD}}^{100}\right) f$. Due to smallness of $\delta_{i} \sim \mathcal{O}\left(m_{\nu}^{4}\right)$, the constraint equations (22) and (23) are easily solved for any values of $\Delta k_{\nu}$ and $T_{\mathrm{QCD}}$. For example, imposing the constraint $\Delta k_{\nu}<0.3$ [17], leads to the bounds

$$
\begin{aligned}
& m_{\nu_{\mu}} \lesssim \begin{cases}170 \mathrm{KeV} & T_{Q C D}=100 \mathrm{MeV} \\
150 \mathrm{KeV} & T_{Q C D}=200 \mathrm{MeV}\end{cases} \\
& m_{\nu_{\tau}} \lesssim \begin{cases}210 \mathrm{KeV} & T_{Q C D}=100 \mathrm{MeV} \\
190 \mathrm{KeV} & T_{Q C D}=200 \mathrm{MeV}\end{cases}
\end{aligned}
$$

Let us finally mention that there were claims recently that the effective number of extra neutrino species is not larger than 0.1 [18]. If this is indeed the case the right-handed neutrinos should decouple before or near electroweak phase transition. Using this bound the limit on both the $\nu_{\tau}$ and $\nu_{\mu}$ masses is, conservatively, $10 \mathrm{KeV}$.

A.D.Dolgov is grateful for hospitality to the Department of Physics of University of Michigan where this work started and to the Astrophysical Group at Fermilab where it was finished. K.K. wishes to thank the Finnish Academy for financial support. This research is supported by the DOE grant DE-AC02-83ER40105. 


\section{References}

[1] S.S. Gerstein and Ya.B. Zeldovich, Zh. Eksp. Teor. Fiz. Pis'ma 4, 174 (1972).

RCowsik and J. McClelland, Phys. Rev. Lett. 29, 669 (1972).

[2] P. Hut, Phys. Lett. 69B, 85 (1977); K. Sato and H. Kobayashi, Prog. Theor. Phys. 58, 1775 (1977); B.W. Lee and S. Weinberg Phys. Rev. Lett. 39, 165 (1977); M.I. Vysotsky, A.D. Dolgov and Ya.B. Zeldovich, JETP Lett. 26, 188 (1977).

[3] K. Assamsan et. al. PSI-PR-94-19.

[4] ARGUS Collab., H. Albrecht et. al., Phys Lett B292, 221 (1992).

[5] E. Kolb and R. Scherrer Phys. Rev. D25, 1481 (1982); E. Kolb, M.S. Turner, A. Chakravorty, and D.N. Schramm Phys. Rev. Lett. 67, 533 (1991).

[6] A.D. Dolgov and I.Z. Rothstein Phys. Rev. Lett. (1993); M. Kawasaki et.al. Nucl. Phys. B419, 105 (1994); S. Dodelson, G. Gyuk and M.S. Turner, Phys. Rev. D49, 5068 (1994).

[7] K. Enqvist and H. Uibo Pys. Lett. B 301 (1993) 376. Some of the most efficient reactions in (1) were significantly underestimated here, leading to somewhat too weak bounds even for an analysis relying solely on the decoupling argument.

[8] G.M. Fuller and R.A. Malaney, Phys. Rev. D43, 3136 (1991).

[9] W.P. Lam and K.-W. Ng, Phys. Rev. D44, 3345 (1991).

[10] R. Mayle, D.N. Schramm, M.S. Turner, and J.R. Wilson, Phys. Lett. B317, 119 (1993).

[11] A. Burrows, R. Ghandi, and M.S. Turner, Phys. Rev. Lett. 68 (1992) 3834.

[12] K.S. Babu, R.N. Mohapatra and I.Z. Rothstein Phys. Rev. Lett. 67, 545 (1991).

[13] K.S. Babu, R.N. Mohapatra and I.Z. Rothstein Phys. Rev. D45, R3312 (1992).

[14] M. Srednicki, R. Watkins and K.A. Olive, Nucl. Phys. B310, 693 (1988). 
[15] E. Fischbach, et.al. Phys. Rev. D16, 2377 (1977).

[16] T. Kalogeropoulous, J Schechter, and J. Valle, Phys. Lett. 86B, 72 (1979).

[17] T. Walker et.al., Astrophys. J. 376, 51 (1991).

[18] K.A. Olive and G. Steigman UMN-TH-1230/94 OSU-TA-6/94 